\title{
An Algorithm to Solve the Partition into Perfect Matchings Problem in Halin Graphs
}

\author{
Yunting Lu and Dingjun Lou \\ Department of Computer Science, Sun Yat-sen University \\ Guangzhou 510275, P.R. China \\ lu-yunting@163.com, issldj@sysu.edu.cn
}

\begin{abstract}
In this paper, we consider whether the vertices of graph $G$ can be partitioned into $K$ subsets $V_{1}, V_{2}, \ldots V_{K}$ so that for each $i \in$ $\{1, \ldots, K\}$, the subgraph induced by $V_{i}$ is a perfect matching where $K \leq$ $|V|$. It is known that it is an NP complete problem in general graphs. In this paper, we restrict the problem in Halin graphs and give an algorithm to find the minimum value of $K(2 \leq K \leq 4)$ in Halin graphs. The time complexity is $O(n)$.
\end{abstract}

Keywords: Halin Graph, Perfect matching, Algorithm.

\section{Introduction and Terminology}

Let $M$ be a matching of graph $G$ and $v \in V(G)$. If $v$ is incident with an edge in $M, v$ is called $M$-saturated. If every vertex of $G$ is $M$-saturated, then $M$ is a perfect matching of $G$. If edges in graph $G$ share a common vertex or adjacent to a same edge, these edges are said to be connected. A perfect matching $M$ in $G$ is called $k$-colored if at least $k$ colors are used to color the edges in $M$ such that any two connected edges in $M$ have different colors, and $k$ is called the chromatic number of $M$. If the minimum chromatic number of all the perfect matchings in graph $G$ is $k, G$ is called $k$ perfect matching colored (PMC).

A Halin graph $H=T \cup C$ is obtained by embedding a tree $T$ having no nodes of degree 2 in the plane, and then add a cycle $C$ to join the leaves of $T$ in such a way that the resulting graph is planar. If $T$ is a star, that is, a single vertex joining to the other vertices, then $H=T \cup C$ is called a wheel. Suppose $T$ has at least two non-leaves. Let $w$ be a non-leaf of $T$ which is adjacent to only one non-leaf of $T$. Then the set of leaves of $T$ adjacent to $w$, which we denote by $C(w)$, comprises a consecutive subsequence of the cycle $C$. We call the subgraph of $H$ induced by $\{w\} \cup C(w)$ a fan and call $w$ the center of the fan. An edge cutset of a connected graph $G=(V, E)$ is a set of edges whose removal leaves a disconnected graph. If it consists of exactly $k$ edges, then we call it a $k$-edge cutset. Given a fan $F$ of $H$, the three edges connecting $V(F)$ to $V(H-F)$ compose a 3-edge cutset of $H$. We denote the 3-edge cutest by $E C_{3}(F)$.

In the following sections, we use $H=T \cup C$ to denote a Halin graph and $K$ to denote the minimum integer satisfying that $V(H)$ can be partitioned into $K$ sets, say $V_{1}, \ldots, V_{K}$, such that $H\left[V_{i}\right]$ is a perfect matching for all $i \in\{1, \ldots, K\}$. 


\section{Preliminary Results}

Lemma 1. The chromatic number of a planar graph is 4.

\section{Main Results}

Theorem 1. Let $H$ and $K$ be as defined as above. And $H$ has even order. Then $2 \leq K \leq 4$.

Proof. Halin graphs have hamilton cycles and if any node is deleted, the resulting graph still has a hamilton cycle 2. So $H$ has a perfect matching. Assume $M$ is a perfect matching in $H$. Let $H^{\prime}$ be a graph by contracting each edges in $M$. Let $e(v)$ be the edge in $M$ such that $v$ is the vertex constructed in $H^{\prime}$ after contracting $e(v)$. Obviously $H^{\prime}$ is planar, so by Lemma $1, H^{\prime}$ is 4-colored. Assume 1, 2, 3, 4 are the four colors used to color $H^{\prime}, W_{i}$ are all the vertices in $H^{\prime}$ which are colored color $i$ and $U_{i}=\left\{e(v): v \in W_{i}\right\}$ where $1 \leq i \leq 4$. Let $e\left(v_{1}\right)$ and $e\left(v_{2}\right)$ be any two edges in $U_{i}$, then $v_{1}, v_{2} \in W_{i}$. Then $e\left(v_{1}\right)$ and $e\left(v_{2}\right)$ are not connected, otherwise $v_{1}$ and $v_{2}$ have different colors, contradicting $v_{1}$, $v_{2} \in W_{i}$. So let $T_{i}=\left\{v: v \in V(e)\right.$ and $\left.e \in U_{i}\right\}$ where $1 \leq i \leq 4$ and then $H\left[T_{i}\right]$ is a matching in $H$. Since $\cup_{i=1}^{4} U_{i}=M$, then $\cup_{i=1}^{4} T_{i}=V(H)$. So $K \leq 4$. $K$ can not be 1 . So $2 \leq K \leq 4$.

Theorem 2. $K=k$ if and only if $H$ is $k P M C$.

Proof. Suppose $K=k$. Assume $H$ is $k^{\prime}$ PMC. Then the vertices of a Halin graph $H$ can be partitioned into $k$ subsets, say $V_{1}, V_{2}, \ldots, V_{k}$, such that the subgraph induced by $V_{i}$ is a perfect matching, say $M_{i}$, where $1 \leq i \leq k$. So $M=M_{1} \cup$ $M_{2} \cup \ldots \cup M_{k}$ is a perfect matching in $H$. Obviously, $M$ is $k$-colored because if edges in $M_{i}$ are colored with a same color $c_{i}$ where $1 \leq i \leq k$ and $c_{x} \neq c_{y}$ (if $x \neq y$ and $1 \leq x, y \leq k)$, then no adjacent edges in $M$ have a same color. So $k^{\prime} \leq k$. Suppose $k^{\prime}<k$. Then there is a perfect matching $M^{\prime}$ in $H$ such that $M^{\prime}$ is $k^{\prime}$-colored. Let $M_{i}$ be all the edges in $M$ that have one same color where $1 \leq i \leq k^{\prime}$. Then $V\left(V_{1}\right) \cup V\left(V_{2}\right) \cup \ldots \cup V\left(V_{k^{\prime}}\right)=V(H), H\left[V_{1}\right]=M_{1}, H\left[V_{2}\right]=M_{2}$ $\ldots$ and $H\left[V_{k^{\prime}}\right]=M_{k^{\prime}}$. The subgraph induced by $V_{i}$ is a perfect matching, so $k \leq k^{\prime}$. A contradiction with $k^{\prime}<k$. That is $k^{\prime}=k, H$ is $k \mathrm{PMC}$.

Now we prove sufficiency. If $H$ is $k$ PMC, then there exists a perfect matching, say $M$, such that $M$ is $k$-colored. Let $M_{i}$ are all edges in $M$ that have one same color where $1 \leq i \leq k$. $V_{1}=V\left(M_{1}\right), V_{2}=V\left(M_{2}\right) \ldots$ and $V_{k}=V\left(M_{k}\right)$. Then $V\left(V_{1}\right) \cup V\left(V_{2}\right) \cup \ldots \cup V\left(V_{k}\right)=V(H)$. The subgraph induced by $V_{i}$ is a perfect matching, so $K \leq k$. Suppose $K<k$, Then the vertices of a Halin graph $H$ can be partitioned into $K$ subsets, say $V_{1}, V_{2}, \ldots, V_{K}$, such that the subgraph induced by $V_{i}$ is a perfect matching, say $M_{i}$, where $1 \leq i \leq K$. So $M=M_{1} \cup M_{2} \cup$. . $\cup M_{K}$ is a perfect matching in $H$. Hence $M$ is $K$-colored, so $k \leq K$. A contradiction with $K<k$. That is $K=k$. So $K=k$ if and only if $H$ is $k$ PMC.

Since all the vertices in $V_{i}$ are colored with the same colors, an edge coloring about the Halin graph $H$ is obtained. It obeys that the colored edges must 
cover all the vertices of $H$ and if two edges are connected, they are colored with different colors. So we convert the problem to the edge coloring in Halin graphs.

\section{Description of the Algorithm}

Firstly we briefly describe the method of shrinking fan firstly appeared in [3]. Let $F$ be a fan. We use $H \times F$ to denote the graph obtained from $H$ by shrinking $F$ to a new "pseudo-vertex", say $v_{F}$; that is, $V(H \times F)=\left\{v_{F}\right\} \cup\{V(H) \backslash V(F)\}$ and the edges of $H \times F$ are defined as follows:

1. An edge with both ends in $F$ is deleted;

2. An edge with both ends in $H-F$ remains unchanged;

3. An edge with one end-vertex in $H-F$ and the other in $F$ now joins the incident vertex of $H-F$ and $v_{F}$.

By Theorem $1, K$ is 2,3 or 4 . Firstly we check whether $K=2$. If $K \neq 2$, check whether $K=3$. If $K \neq 3, K=4$. We discuss it in the following two cases.

\subsection{Case 1: Check Whether $K=2$}

By Theorem 2, in order to check whether $K=2$, we only need to check whether there is a perfect matching in $H$ which is 2-colored. Let $F$ be a fan of $H, M$ be a perfect matching of $H$ and $E C_{3}(F)=\{k, j, l\}$. Let $k=u_{k} v_{k}, j=u_{j} v_{j}$ and $l=u_{l} v_{l}$ such that $u_{k}, u_{j}, u_{l} \in V(F), v_{k}, v_{j}, v_{l} \in V(H-F), j, l \in C$ and the direction $v_{j}, u_{j}, u_{l}, v_{l}$ is anti-clockwise in the cycle $C$.

Since $M$ is a perfect matching of $H$, each vertex of $H$ is saturated by $M$. If $k \in M$, we say the vertex $u_{k}$ is externally saturated in $F$; otherwise, internally. The denotations are the same to $u_{j}$ and $u_{l}$. Only the situation of $\left\{u_{k}\right.$, $\left.u_{j}, u_{l}\right\}$ affects the situation of $M \cap(H-F)$. In the following we use $\operatorname{Pos}(v)$ to denote whether the vertex $v$ is internally or externally colored where $\operatorname{Pos}(v) \in$ $\{$ externally, internally\}, Color $(v)$ to denote which color vertex $v$ is colored where $\operatorname{Color}(v) \in\left\{c_{1}, c_{2}\right\}$, and $X(v)$ to denote the coloring state of vertex $v$. We define the following four situations respectively:

$$
\begin{aligned}
& X(v)=1 \text { means } \operatorname{Pos}(v)=\text { internally and } \operatorname{Color}(v)=c_{1} ; \\
& X(v)=2 \text { means } \operatorname{Pos}(v)=\text { externally and } \operatorname{Color}(v)=c_{1} ; \\
& X(v)=3 \text { means } \operatorname{Pos}(v)=\text { internally and } \operatorname{Color}(v)=c_{2} ; \\
& X(v)=4 \text { means } \operatorname{Pos}(v)=\text { externally and } \operatorname{Color}(v)=c_{2} ;
\end{aligned}
$$

We use a triple $\left(X\left(u_{k}\right), X\left(u_{j}\right), X\left(u_{l}\right)\right)$ to denote one situation of $u_{k}, u_{j}, u_{l}$. And $T(F)$ is a set of all the triples to $F$ in all the colorings. After shrinking a fan $F$ to a pseudo vertex $v_{F}$, we set $T\left(v_{F}\right)=T(F)$.

We regard an original vertex on the cycle $C$ as a special fan. Let $C$ be the cycle of $H$ and $u$ be an original vertex on $C$. Let $j=u v_{j}, l=u v_{l}$ and $k=$ $u v_{k}$ such that $v_{j}, v_{l} \in V(C), v_{k} \in V(H-C)$ and the direction of $v_{j}, u, v_{l}$ is anti-clockwise in the cycle $C$. If $k \in M$, we say the vertex $u$ is externally 


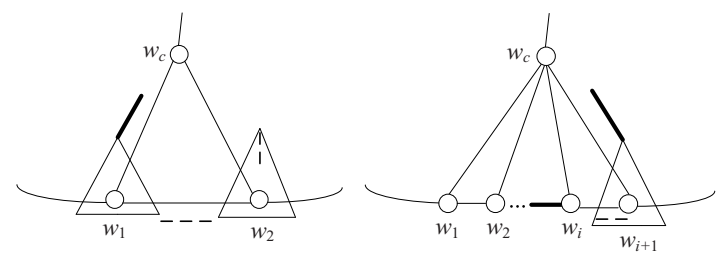

Fig. 1. Two examples of computing $T\left(P F_{2}\right)$ and $T\left(P F_{i}\right)$ (Thick lines are colored color $c_{1}$ and broken lines are colored color $c_{2}$ )

saturated and use a triple $(X(u), 0,0)$ where $X(u)=2$ or 4 to denote a possible situation. If $j \in M$, we use a triple $(0, X(u), 0)$ to denote this situation. If $l \in M$, similarly. So for each leaf $v$ in $H$, we relate $v$ with a set of triples $T(v)=\{(2,0,0),(4,0,0),(0,2,0),(0,4,0),(0,0,2),(0,0,4)\}$.

Now we describe how to get all the triples in a certain fan $F$. Dynamic programming is used here. Let $F$ be a fan of $H$. Let $w_{c}$ be the center of $F$ and $w_{1}, w_{2} \ldots w_{r}$ be the vertices on the cycle $C$ in anti-clockwise order. Each $w_{i}$ $(1 \leq i \leq r)$ can be either an original vertex or a pseudo-vertex. We denote the induced subgraph $H\left[\left\{w_{c}, w_{1}, w_{2}, \ldots, w_{k}\right\}\right]$ by pseudo-fan $P F_{k}$ of $F(2 \leq k \leq r)$. Note that if $w_{c} w_{i} \in M(k+1 \leq i \leq r)$, then vertex $w_{c}$ is said to be externally saturated according to the pseudo-fan $P F_{k}$ in $M$. Hence $T\left(P F_{i}\right)$ is a set of all the triples to the pseudo-fan $P F_{i}$. We calculate the situation of $k=2$ first.

$$
\begin{aligned}
& T\left(P F_{2}\right)=\left\{t_{1} \bullet s_{1} \mid t_{1} \bullet s_{1} \neq N U L L \text { and } t_{1} \in T\left(w_{1}\right) \text { and } s_{1} \in T\left(w_{2}\right)\right\} . \\
& \text { Let } y, z \in\{1,2,3,4\} \cdot t_{1} \bullet s_{1}=N U L L \text { except the following situations: } \\
& (2, y, 1) \bullet(3,3, z)=(1, y, z),(2, y, 3) \bullet(3,1, z)=(1, y, z),(2, y, 2) \bullet(3,2, z)=(1, y, z) \\
& (2, y, 4) \bullet(3,4, z)=(1, y, z),(3, y, 1) \bullet(2,3, z)=(1, y, z),(3, y, 3) \bullet(2,1, z)=(1, y, z) \\
& (3, y, 2) \bullet(2,2, z)=(1, y, z),(3, y, 4) \bullet(2,4, z)=(1, y, z),(2,0,0) \bullet(0,0,4)=(1,1,4) \\
& (0,4,0) \bullet(2,0,0)=(1,4,1),(2,0,0) \bullet(3,3, z)=(1,1, z),(3, y, 3) \bullet(2,0,0)=(1, y, 1) \\
& (3, y, 1) \bullet(3,3, z)=(2, y, z),(3, y, 3) \bullet(3,1, z)=(2, y, z),(3, y, 2) \bullet(3,2, z)=(2, y, z) \\
& (3, y, 4) \bullet(3,4, z)=(2, y, z),(0,4,0) \bullet(3,1, z)=(2,4, z),(0,0,4) \bullet(3,4, z)=(2,3, z) \\
& (3, y, 1) \bullet(0,0,4)=(2, y, 4),(3, y, 4) \bullet(0,4,0)=(2, y, 3),(0,0,4) \bullet(0,4,0)=(2,3,3) \\
& (4, y, 3) \bullet(1,1, z)=(3, y, z),(4, y, 1) \bullet(1,3, z)=(3, y, z),(4, y, 4) \bullet(1,4, z)=(3, y, z) \\
& (4, y, 2) \bullet(1,2, z)=(3, y, z),(1, y, 3) \bullet(4,1, z)=(3, y, z),(1, y, 1) \bullet(4,3, z)=(3, y, z) \\
& (1, y, 4) \bullet(4,4, z)=(3, y, z),(1, y, 2) \bullet(4,2, z)=(3, y, z),(4,0,0) \bullet(0,0,2)=(3,3,2) \\
& (0,2,0) \bullet(4,0,0)=(3,2,3),(4,0,0) \bullet(1,1, z)=(3,3, z),(1, y, 1) \bullet(4,0,0)=(3, y, 3) \\
& (1, y, 3) \bullet(1,1, z)=(4, y, z),(1, y, 1) \bullet(1,3, z)=(4, y, z),(1, y, 4) \bullet(1,4, z)=(4, y, z) \\
& (1, y, 2) \bullet(1,2, z)=(4, y, z),(0,2,0) \bullet(1,3, z)=(4,2, z),(0,0,2) \bullet(1,2, z)=(4,1, z) \\
& (1, y, 3) \bullet(0,0,2)=(4, y, 2),(1, y, 2) \bullet(0,2,0)=(4, y, 1),(0,0,2) \bullet(0,2,0)=(4,1,1)
\end{aligned}
$$

Suppose $w_{1}, w_{2}$ are pseudo vertices. Let $u_{c}$ (the center), $u_{1}, u_{2} \ldots u_{r}$ be all the vertices in $H$ after restoring the pseudo vertex in $w_{1}$ and $u_{c}^{\prime}$ (the center), $u_{1}^{\prime}, u_{2}^{\prime} \ldots u_{r}^{\prime}$ be all the vertices in $H$ after restoring the pseudo vertex in $w_{2}$. If $\left(X\left(u_{c}\right), X\left(u_{1}\right), X\left(u_{r}\right)\right)=(2, y, 4)$ and $\left(X\left(u_{c}^{\prime}\right), X\left(u_{1}^{\prime}\right), X\left(u_{r}^{\prime}\right)\right)=(3,4, z)$, then 
the triple $\left(X\left(w_{c}\right), X\left(w_{1}\right), X\left(w_{2}\right)\right)$ in $T\left(P F_{2}\right)$ equals $(1, y, z)$, that is $(2, y, 4) \bullet(3$, $4, z)=(1, y, z)$. See Fig.1 (The left).

Once we have the set $T\left(P F_{i}\right)$, we can calculate $T\left(P F_{i+1}\right)$.

$T\left(P F_{i+1}\right)=\left\{t_{1} \bullet s_{1} \mid t_{1} \bullet s_{1} \neq N U L L\right.$ and $t_{1} \in T\left(P F_{i}\right)$ and $\left.s_{1} \in T\left(w_{i+1}\right)\right\}$ $(2 \leq i \leq r-1)$, Similarly, we present the formulas to the calculation as following:

$$
\begin{aligned}
& t_{1} \bullet s_{1}=N U L L \text { except the following situations: } \\
& (1, y, 1) \bullet(3,3, z)=(1, y, z),(1, y, 3) \bullet(3,1, z)=(1, y, z),(1, y, 2) \bullet(3,2, z)=(1, y, z) \\
& (1, y, 4) \bullet(3,4, z)=(1, y, z),(1, y, 1) \bullet(0,0,4)=(1, y, 4),(1, y, 4) \bullet(0,4,0)=(1, y, 3) \\
& (3, y, 3) \bullet(1,1, z)=(3, y, z),(3, y, 1) \bullet(1,3, z)=(3, y, z),(3, y, 4) \bullet(1,4, z)=(3, y, z) \\
& (3, y, 2) \bullet(1,2, z)=(3, y, z),(3, y, 3) \bullet(0,0,2)=(3, y, 2),(3, y, 2) \bullet(0,2,0)=(3, y, 1) \\
& (2, y, 1) \bullet(2,3, z)=(1, y, z),(2, y, 3) \bullet(2,1, z)=(1, y, z),(2, y, 2) \bullet(2,2, z)=(1, y, z) \\
& (2, y, 4) \bullet(2,4, z)=(1, y, z),(2, y, 3) \bullet(2,0,0)=(1, y, 1) \\
& (4, y, 3) \bullet(4,1, z)=(3, y, z),(4, y, 1) \bullet(4,3, z)=(3, y, z),(4, y, 4) \bullet(4,4, z)=(3, y, z) \\
& (4, y, 2) \bullet(4,2, z)=(3, y, z),(4, y, 1) \bullet(4,0,0)=(3, y, 3) \\
& (2, y, 1) \bullet(3,3, z)=(2, y, z),(2, y, 3) \bullet(3,1, z)=(2, y, z),(2, y, 2) \bullet(3,2, z)=(2, y, z) \\
& (2, y, 4) \bullet(3,4, z)=(2, y, z),(2, y, 1) \bullet(0,0,4)=(2, y, 4),(2, y, 4) \bullet(0,4,0)=(2, y, 3) \\
& (4, y, 3) \bullet(1,1, z)=(4, y, z),(4, y, 1) \bullet(1,3, z)=(4, y, z),(4, y, 4) \bullet(1,4, z)=(4, y, z) \\
& (4, y, 2) \bullet(1,2, z)=(4, y, z),(4, y, 3) \bullet(0,0,2)=(4, y, 2),(4, y, 2) \bullet(0,2,0)=(4, y, 3) \\
& \text { Once the set } T\left(P F_{r}\right) \text { is obtained, we can calculate } T(F)=T\left(P F_{r}\right) \text {. }
\end{aligned}
$$

Suppose $w_{i+1}$ is a pseudo vertex. Let $u_{c}$ (the center), $u_{1}, u_{2} \ldots u_{r}$ be all the vertices in $H$ after restoring the pseudo vertex in $w_{i+1}$. If one triple in $T\left(P F_{i}\right)$ is $\left(X\left(w_{c}\right), X\left(w_{1}\right), X\left(w_{i}\right)\right)=(2, y, 1)$ and $\left(X\left(u_{c}\right), X\left(u_{1}\right), X\left(u_{r}\right)\right)=(2,3, z)$, then the triple $\left(X\left(w_{c}\right), X\left(w_{1}\right), X\left(w_{i+1}\right)\right)$ in $T\left(P F_{i+1}\right)$ equals $(1, y, z)$, that is $(2, y, 1) \bullet(2,3, z)=(1, y, z)$. See Fig.1 (The right).

Firstly, our algorithm picks a non-leaf vertex $r$ as the root of $T$ such that $r$ is adjacent to a leaf $f$ of $T$ and perform a postorder scan of $T$. When a vertex is scanned there are three possibilities: (I) $v$ is a leaf, it is simply bypassed, (II) $v$ is a non-leaf different from $r, v$ and all its children constitute a fan $F$. We shrink $F$ to $v_{F}$ and get $T(F)$. Let $T\left(v_{F}\right)=T(F)$, (III) $v=r$, then $H$ has been reduced to a wheel. Let $F \equiv H-f$. We consider $F$ as a fan and shrink $F$ to a pseudo vertex $r^{\prime}$ and get $T\left(r^{\prime}\right)=T\left(P F_{k-1}\right)$, then $H$ is reduced to a graph as Fig.2.

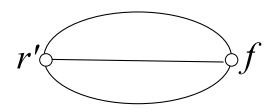

Fig. 2. The graph after shrinking all fans in $H$

$T\left(r^{\prime}\right)$ and $T(f)$ can match if and only if there is a triple $(x, y, z)$ in $T\left(r^{\prime}\right)$ and a triple $\left(x^{\prime}, y^{\prime}, z^{\prime}\right)$ in $T(f)$ such that they can match in a legal 2 coloring, that is, they satisfy the following conditions:

(1) If $x^{\prime}=2$, then $x=2, y=z=3$ else if $x^{\prime}=4, x=4, y=z=1$.

(2) If $y^{\prime}=2$, then $z=2, x=y=3$ else if $y^{\prime}=4, z=4, x=y=1$.

(3) If $z^{\prime}=2$, then $y=2, x=z=3$ else if $z^{\prime}=4, y=4, x=z=1$. 
Theorem 3. Let $r^{\prime}$ be the vertex after applying the shrinking operations on fans in $H$ recursively and $T\left(r^{\prime}\right), T(f)$ as defined above. Then $T\left(r^{\prime}\right)$ and $T(f)$ can match if and only if $H$ is $2 P M C$.

Proof. To prove this theorem, it is sufficient to prove the following claim.

Claim. Let $H$ be a Halin graph and $F$ be a fan in $H$. Let $w_{c}$ be the center of $F$ and let $w_{1}, w_{2} \ldots w_{r}$ for $r \geq 2$ be the vertices of $F$ belong to $C . T\left(P F_{i}\right)$ includes all the possible triples in a pseudo fan $P F_{i}$ where $2 \leq i \leq r$.

Proof. It is sufficient to prove $T\left(P F_{i}\right)$ includes all the possible triples in a pseudo fan $P F_{i}$ where $2 \leq i \leq r$. We use induction on $i$.

(1) When $i=2$, the table $T\left(P F_{2}\right)$ includes all the possible triples according to $T\left(w_{1}\right)$ and $T\left(w_{2}\right)$.

(2)When $i<m$, suppose Claim holds.

(3) When $i=m$, suppose $T\left(P F_{i}\right)$ does not include all the possible triples, that is there exits one triple $\left(X\left(w_{c}\right), X\left(w_{1}\right), X\left(w_{i}\right)\right)$ is not in $T\left(P F_{i}\right)$, but $\left(X\left(w_{c}\right)\right.$, $\left.X\left(w_{1}\right), X\left(w_{i}\right)\right)$ is one situation of $w_{c}, w_{1}, w_{i}$ in a fan $P F_{i}$ actually.

(3.1) If $w_{i}$ is an original vertex

(3.1.1) $(x, 0,0) \in T\left(w_{i}\right), x \in\{2,4\}$.

By the structure of $T\left(P F_{m-1}\right)$, we know it must include the triple $\left(X\left(w_{c}\right)^{\prime}\right.$, $\left.X\left(w_{1}\right), X\left(w_{i-1}\right)\right)$ such that $X\left(w_{i-1}\right)$ MOD $2=1, X\left(w_{i-1}\right) \neq X\left(w_{i}\right), X\left(w_{c}\right)^{\prime}=x$ and $X\left(w_{c}\right)^{\prime}=X\left(w_{i}\right)+1$. Then after $\left(X\left(w_{c}\right)^{\prime}, X\left(w_{1}\right), X\left(w_{i-1}\right)\right) \bullet(x, 0,0)$ we can get the triple $\left(X\left(w_{c}\right), X\left(w_{1}\right), X\left(w_{i}\right)\right)$ in $T\left(P F_{i}\right)$. A contradiction.

(3.1.2) $(0, y, 0) \in T\left(w_{i}\right), y \in\{2,4\}$. Similarly.

(3.1.3) $(0,0, z) \in T\left(w_{i}\right), z \in\{2,4\}$. Similarly.

(3.2) If $w_{i}$ is a pseudo-vertex. Suppose we get $w_{i}$ after shrinking $F$, let $v_{0}$ be the center of $F$ and $v_{1}, v_{2} \ldots v_{m}$ be the vertices on the cycle $C$ in that order. $\forall(x$, $y, z) \in T\left(w_{i}\right),(x, y, z)$ is equal to $\left(X\left(v_{0}\right), X\left(v_{1}\right), X\left(v_{m}\right)\right), x, y, z \in\{1,2,3,4\}$ (3.2.1) If $\operatorname{Pos}\left(v_{0}\right)=$ externally and $\operatorname{Pos}\left(v_{1}\right)=$ externally

By the structure of $T\left(P F_{m-1}\right)$, it must include one triple $\left(X\left(w_{c}\right)^{\prime}, X\left(w_{1}\right)\right.$, $\left.X\left(w_{i-1}\right)\right)$ such that $X\left(w_{c}\right)^{\prime}=X\left(w_{c}\right)+1=X\left(v_{0}\right)$ and $X\left(w_{i-1}\right)=X\left(v_{1}\right)$. Then after $\left(X\left(w_{c}\right)^{\prime}, X\left(w_{1}\right), X\left(w_{i-1}\right)\right) \bullet(x, y, z)$ we can get the triple $\left(X\left(w_{c}\right), X\left(w_{1}\right)\right.$, $\left.X\left(w_{i}\right)\right)$ in $T\left(P F_{i}\right)$. A contradiction.

(3.2.2) If $\operatorname{Pos}\left(v_{0}\right)=$ externally and $\operatorname{Pos}\left(v_{1}\right)=$ internally. Similarly.

(3.2.3) If $\operatorname{Pos}\left(v_{0}\right)=$ internally and $\operatorname{Pos}\left(v_{1}\right)=$ externally. Similarly.

(3.2.4) If $\operatorname{Pos}\left(v_{0}\right)=$ internally and $\operatorname{Pos}\left(v_{1}\right)=$ internally. Similarly.

So when $i=m, T\left(P F_{i}\right)=T(F)$ includes all the possible triples.

After applying shrinking operations recursively, $H$ is reduced to the graph in Fig.2. Let $V_{1}=$ all vertices in $H$ after restoring each pseudo vertex in $\left.r^{\prime}\right\}, V_{2}=$ $\{f\},\left\{e_{1}, e_{2}, e_{3}\right\}$ are the three edges joining $V_{1}$ to $V_{2}, H_{1}$ and $H_{2}$ are subgraphs constructed by adding $\left\{e_{1}, e_{2}, e_{3}\right\}$ to subgraphs $H\left[V_{1}\right]$ and $H\left[V_{2}\right]$ respectively. Then each $t \in T\left(r^{\prime}\right)$ corresponds a legal 2 coloring of a matching in $H_{1}$ covering all the vertices in $V_{1}$ and so does each $s \in T(f)$. By Claim, $T\left(r^{\prime}\right)$ contains all the possible coloring situations in $H_{1}$ and $T(f)$ contains all the possible coloring situations in $H_{2}$. Then by definition $T\left(r^{\prime}\right)$ and $T(f)$ can match if and only if 
there exists a triple $t$ in $T\left(r^{\prime}\right)$ and a triple $s$ in $T(f)$ such that the coloring in $H_{1}$ that $t$ corresponds and the coloring in $\mathrm{H}_{2}$ that $s$ corresponds can match together in a legal 2 coloring of $H$. So if $T\left(r^{\prime}\right)$ and $T(f)$ can match, $H$ is 2 PMC.

Now we prove sufficiency. Suppose $H$ is $2 \mathrm{PMC}$, there must exist $C$ which is a legal 2 coloring of $H$. $C$ contains a legal 2-coloring $C_{1}$ of $H_{1}$ and a legal 2-coloring $C_{2}$ of $H_{2}$. By Claim, $C_{1}$ corresponds a triple $t$ in $T\left(r^{\prime}\right)$ and $C_{2}$ corresponds a triple $s$ in $T(f)$. Hence $t$ and $s$ can match together in a legal 2 perfect matching coloring of $H$. By definition, $T\left(r^{\prime}\right)$ and $T(f)$ can match. Thus the Theorem holds.

\subsection{Case 2: Check Whether $K=3$}

Similar to Case 1.

\section{Procedure of the Algorithm}

Algorithm to Solve the partition into Perfect Matchings problem in Halin Graphs $K(H)$ 1. Choose a non-leaf vertex of $T$, denoted by $v_{\text {root }}$, such that $v_{\text {root }}$ is adjacent to a leaf of $T$, denoted by $v_{\text {leaf }}$.

2. Perform a postorder scan of $T$, for each fan $F$ has been found do

3 . If the centre of $F \neq v_{\text {root }}$ then

begin

calculate $T(F)$ as Case 1 , shrink $F$ to $v_{F}$, let $T\left(v_{F}\right)=T(F)$; end

Let $H_{w}$ be the wheel we finally get and $k$ be the edge joining $v_{\text {root }}$ and $v_{\text {leaf }}$.

Let $j, l$ be the two edges in $C$ adjacent to $v_{l e a f}$ such that the direction of $j, v_{\text {leaf }}, l$ is anti-clockwise. Let $F_{w} \equiv H_{w}-v_{\text {leaf }}$.

4. Calculate the set $T\left(F_{w}\right)$.

5. Shrink the fan $F_{w}$ to the pseudo-vertex $v_{w}$, let $T\left(v_{w}\right)=T\left(F_{w}\right)$.

6. If $T\left(v_{w}\right)$ and $T\left(v_{\text {leaf }}\right)$ can match.

7. return $K=2$

8. else

9. Perform a postorder scan of $T$, for each fan $F$ has been found do

10. If the centre of $F \neq v_{\text {root }}$ then

begin

calculate $T(F)$ as Case 2 , shrink $F$ to $v_{F}$, let $T\left(v_{F}\right)=T(F)$; end

Let $H_{w}$ be the wheel we finally get and $k$ be the edge joining $v_{\text {root }}$ and $v_{\text {leaf }}$.

Let $j, l$ be the two edges in $C$ adjacent to $v_{\text {leaf }}$ such that the direction of $j, v_{\text {leaf }}, l$ is anti-clockwise. Let $F_{w} \equiv H_{w}-v_{l e a f}$.

11. Calculate the set $T\left(F_{w}\right)$.

12. Shrink the fan $F_{w}$ to the pseudo-vertex $v_{w}$, let $T\left(v_{w}\right)=T\left(F_{w}\right)$.

13. If $T\left(v_{w}\right)$ and $T\left(v_{\text {leaf }}\right)$ can match.

14. return $K=3$

15. else

16. return $K=4$

17. end

18. end 


\section{An Example}

We apply the algorithm to a given Halin graph. Illustrated in Fig.3. $v_{\text {root }}=a$. $(2,3,3)$ in $T\left(a^{\prime}\right)$ and $(2,0,0)$ in $T(l)$ can match, so $K=2$.

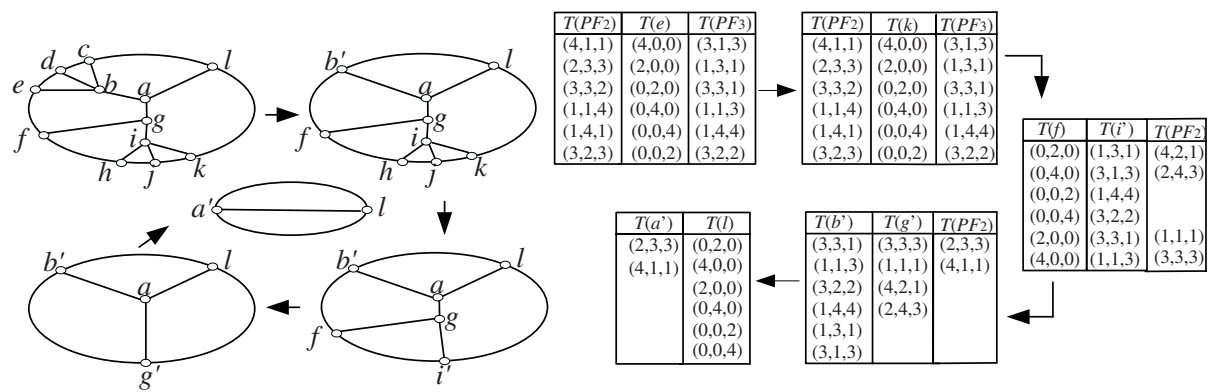

Fig. 3. The procedure of the calculation

\section{The Correctness and the Time Complexity}

Theorem 4. The algorithm $K(H)$ returns $K$.

Proof. The algorithm firstly checks whether $K$ equals 2 . In step 2), it finds a fan $F$. In step 3), it returns all the possible triples to $F$ by Theorem 3. After applying shrinking operations repeatedly, $H$ is reduced to a graph as Fig.2. In the algorithm, if $T\left(r^{\prime}\right)$ and $T(f)$ can match, it returns 2 . So it returns the right $K$ by Theorem 3 . If they cannot match, the algorithm checks whether $K$ equals 3 from 9)-14). Similar to Case 1, if $T\left(r^{\prime}\right)$ and $T(f)$ can match, it returns 3. Otherwise, $K(H)$ returns 4 .

In the algorithm, step 1) needs $O(1)$ time. Step 3) is the operation of shrinking fans in $H$. If a fan $F$ contains $r+1$ vertices, then it is verified that the time of the shrinking operation is $O(r)$. Moreover, shrinking $F$ reduces the number of vertices of the graph by $r$. Thus, the total time of the shrinking operation is $O(|V|)$. The time of the postorder scan without shrinking is bounded by $O(|V|)$. The steps 4)-7) need $O(1)$ time. The time of steps 9)-14) is the same as the steps $2)-7)$. Therefore, the total time for this algorithm is $O(|V|)$.

\section{References}

1. Bondy, J.A., Murty, U.S.: Graph Theory with Applications.1976

2. Bondy, J.A.: Pancyclic Graphs: Recent Results. Colloq. Math. Soc. Janos Bolyai. 10(1975) 181-187

3. Cornuejols, G., Naddef, D., Pulleyblank, W.: Halin Graphs and the Travelling Salesman Problem. Mathematical programming. 26(1983) 287-294 
4. Horton, S.B., Parker, R.G.: On Halin Subgraphs and Supergraphs. Discrete Applied Mathematics. 56(1995) 19-35

5. Lou, D.: Hamiltonian Paths in Halin graphs. Chinese Mathematica Applicata. 8(1995) 158-160 